\title{
Chaos-induced modulation of reliability boosts output firing rate in downstream cortical areas
}

\author{
P. H. E. Tiesinga \\ Department of Physics \& Astronomy, University of North Carolina, Chapel Hill, North Carolina 27599, USA
}

(Received 2 June 2003; published 31 March 2004)

\begin{abstract}
The reproducibility of neural spike train responses to an identical stimulus across different presentations (trials) has been studied extensively. Reliability, the degree of reproducibility of spike trains, was found to depend in part on the amplitude and frequency content of the stimulus [J. Hunter and J. Milton, J. Neurophysiol. 90, 387 (2003)]. The responses across different trials can sometimes be interpreted as the response of an ensemble of similar neurons to a single stimulus presentation. How does the reliability of the activity of neural ensembles affect information transmission between different cortical areas? We studied a model neural system consisting of two ensembles of neurons with Hodgkin-Huxley-type channels. The first ensemble was driven by an injected sinusoidal current that oscillated in the gamma-frequency range $(40 \mathrm{~Hz})$ and its output spike trains in turn drove the second ensemble by fast excitatory synaptic potentials with short term depression. We determined the relationship between the reliability of the first ensemble and the response of the second ensemble. In our paradigm the neurons in the first ensemble were initially in a chaotic state with unreliable and imprecise spike trains. The neurons became entrained to the oscillation and responded reliably when the stimulus power was increased by less than $10 \%$. The firing rate of the first ensemble increased by $30 \%$, whereas that of the second ensemble could increase by an order of magnitude. We also determined the response of the second ensemble when its input spike trains, which had non-Poisson statistics, were replaced by an equivalent ensemble of Poisson spike trains. The resulting output spike trains were significantly different from the original response, as assessed by the metric introduced by Victor and Purpura [J. Neurophysiol. 76, 1310 (1996)]. These results are a proof of principle that weak temporal modulations in the power of gammafrequency oscillations in a given cortical area can strongly affect firing rate responses downstream by way of reliability in spite of rather modest changes in firing rate in the originating area.
\end{abstract}

DOI: 10.1103/PhysRevE.69.031912

PACS number(s): 87.19.La, 87.19.Dd, 87.19.Nn

\section{INTRODUCTION}

The reproducibility of neural spike train responses to an identical stimulus across different trials has recently been studied using a large number of different experimental preparations and stimulus ensembles [1-9]. When the neuron is driven by a fluctuating stimulus the spike trains are often similar across trials, leading to peaks of elevated firing frequency - events - in the spike time histogram [10-17]. A variety of measures have been used to quantify the degree of reproducibility of spike trains $[4,11,13,17,18]$. There are three different aspects of reproducibility, two of which can be quantified using only the spike time histogram. The reliability of an event is the fraction of trials during which a spike is obtained during an event, the precision is the inverse of the jitter in spike times during an event [11]. The third measure derives from non-Poisson statistics due to spike patterns in trains of neural discharge $[18,19]$. The attractor reliability is a measure of the robustness of these patterns and cannot be determined using only the spike time histogram. Indeed, it quantifies the deviation of the spike train statistics from Poisson statistics [18]. The responses across different trials can sometimes be interpreted as the response of an ensemble of similar neurons to one stimulus presentation [20]. Reliability then corresponds to the degree of synchrony between different neurons.

Localized synchronous oscillations in the gammafrequency range $(30-80 \mathrm{~Hz})$ have been observed using human electroencephalographic recordings under various behavioral conditions $[21,22]$ and may be under control of neuromodulators (see Ref. [23] and references therein). In macaque monkeys attention modulated the degree of coherence in the gamma-frequency range between neurons in cortical area V4 and the local field potential (LFP) [24,25]. The reliability of neurons injected by oscillating currents has been studied experimentally $[12-15,17,26]$. For small driving amplitudes (compared with the intrinsic noise level) the discharge was unreliable, whereas for large amplitudes it was reliable across a large frequency range. However, for intermediate amplitudes, reliability depended on driving frequency: it was enhanced when neurons were phase locked [17]. These results predict that reliability in vivo can be modulated by the presence of synchronous oscillations. The question then is, what would be the downstream effect of these reliability modulations?

We consider the information transmission between two model cortical areas, each represented by an ensemble of neurons (Fig. 1). Our goal is to determine the following: (1) the relationship between the reliability of the first ensemble and the firing rate of the second ensemble; and (2) to what extent (non-Poisson) spike patterns in the output spike trains of the first ensemble affect the response of the second ensemble. The model neurons had Hodgkin-Huxley-type voltage-gated channels and were connected by fast excitatory synapses with short term depression (STD, see the Methods). The first ensemble was driven by an injected current that oscillated in the gamma-frequency range and its output spike trains in turn drove the second ensemble. The oscillating current represented the inputs of neurons that were synchronized in the gamma-frequency range. Their activity corre- 


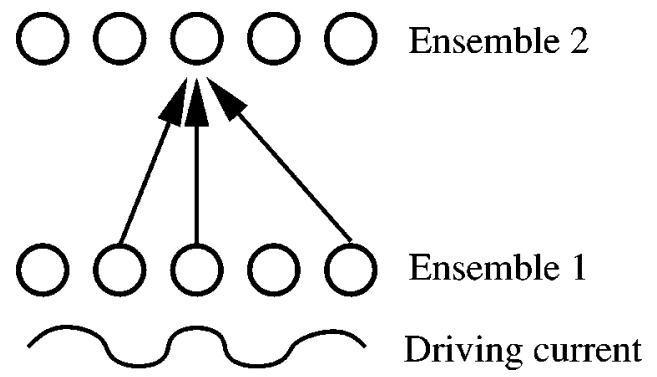

FIG. 1. Diagram of the network geometry. The first ensemble consisted of $N=500$ neurons, each driven by a sinusoidal current. The second ensemble also consisted of $N=500$ neurons. Each of them received AMPAergic synaptic inputs from a different set of $N_{\text {con }}=100$ neurons chosen randomly from the first ensemble. The neurons in ensembles 1 and 2 were not connected among themselves. There were also no feedback connections from ensemble 2 to ensemble 1.

sponds to what would be measured as oscillations in the local field potential [24]. In experiments on behaving macaque monkeys, the power in the gamma-frequency range of the LFP displayed transient increases [24,25]. In our simulations these were modeled as increases in the amplitude of the oscillatory current. Our results are as follows: A small, transient increase in gamma power led to a modest increase in the firing rate of the first ensemble together with an increased reliability and precision. However, the firing rate of the second ensemble increased even more. The spike trains of the first ensemble had non-Poisson statistics because of the presence of spike patterns. When the input spike trains to the second ensemble were replaced by an equivalent input with Poisson statistics, the strong increase in firing rate of the second ensemble was still obtained, but the output spike trains were significantly different.

\section{METHODS}

\section{A. Neuron model}

The model consisted of two ensembles, each with $N$ $=500$ neurons (Fig. 1). Each neuron had a fast transient sodium current $I_{N a}$, a delayed rectifier potassium current $I_{K}$, and a leak current $I_{L}$ and was injected by a time-varying current, $I(t)=I_{0}+I_{A} \cos 2 \pi f_{d} t$, and a white noise current $C_{m} \eta$. The mean of $\eta$ was zero, $\langle\eta(t)\rangle=0$, and the variance was $2 D,\left\langle\eta(t) \eta\left(t^{\prime}\right)\right\rangle=2 D \delta\left(t-t^{\prime}\right)$. Here $\langle\cdot\rangle$ is the average over the noise ensemble and $C_{m}=1 \mu \mathrm{F} / \mathrm{cm}^{2}$ is the membrane capacitance (normalized by area). The driving frequency was $f_{d}=40 \mathrm{~Hz}$. The equation for the membrane potential $V$ reads

$$
C_{m} \frac{d V}{d t}=-I_{N a}-I_{K}-I_{L}+I(t)+C_{m} \eta(t) .
$$

The model equations for the single neuron and implementation are exactly as described in Ref. [27] and are not repeated here. The model used here was adapted from that introduced by Wang and Buzsaki [28].
Each neuron in the second ensemble received inputs from $N_{\text {con }}=100$ neurons from the first ensemble. $N$ represents the number of similar neurons receiving approximately the same inputs, whereas $N_{\text {con }}$ is the number of these neurons that project to one given downstream neuron. The appropriateness of our choice of $N$ and $N_{\text {con }}$ values in relation to data on cortical circuits will be discussed in the Discussion. The neurons were connected by $\alpha$-amino-3-hydroxy-5methyl-4-isooxazolepropionic acid (AMPA) synapses [29]. The unitary strength of synaptic inputs is history dependent on multiple time scales [30]. STD reduces the synaptic strength when the presynaptic neuron produces spikes at a high rate. A number of computational roles have been proposed for STD: it can remove redundancy in spike trains [31] and provide cortical gain control [32]. Here we use the model for STD that is given in Ref. [32]. Each spike from a neuron in the first ensemble led to a current pulse $\delta g d(t) e^{-t / \tau}\left(V-E_{\mathrm{AMPA}}\right)$ in the postsynaptic neuron. Here $\tau=3 \mathrm{~ms}$ [29] was the synaptic decay constant, $d(t)$ was the depression variable, $\delta g=g_{\max } / N_{\text {con }}$ was the unitary conductance, $t$ was the time since the arrival of the presynaptic spike, and $E_{\mathrm{AMPA}}=0 \mathrm{mV}$ is the reversal potential. The maximum synaptic conductance was $g_{\max }=0.1 \mathrm{mS} / \mathrm{cm}^{2}$ for simulations with STD and $g_{\max }=0.03 \mathrm{mS} / \mathrm{cm}^{2}$ for those without STD. Each time a presynaptic neuron fired, $d$ was reduced by a factor 0.75 after the spike was transmitted. Between two presynaptic spikes $d(t)$ approached unity exponentially with a time constant equal to $300 \mathrm{~ms}$ [32].

The standard set of parameters was, for the first ensemble, $I_{0}=0.0 \mu \mathrm{A} / \mathrm{cm}^{2}, D=0.004 \mathrm{mV} / \mathrm{ms}^{2}$, and $I_{A}=2.08 \mu \mathrm{A} / \mathrm{cm}^{2}$ (chaotic state) or $I_{A}=2.30 \mu \mathrm{A} / \mathrm{cm}^{2}$ (phase-locked state). For the second ensemble, $I_{0}=-0.3 \mu \mathrm{A} / \mathrm{cm}^{2}, D=0.04 \mathrm{mV}^{2} / \mathrm{ms}$ and $I_{A}=0$.

\section{B. Statistical analysis}

General notation. Spike times were calculated as the time that the membrane potential $[V$ in Eq. (1)] crossed $0 \mathrm{mV}$ from below. $t_{n i}$ is the $i$ th spike time by the $n$th neuron, likewise $t_{m j}$ is the $j$ th spike time by the $m$ th neuron. Since neurons within an ensemble are not coupled, $t_{n i}$ could equivalently be considered as the $i$ th spike during the $n$th trial. $N$ is the total number of neurons in the ensemble (or the total number of trials), $N_{s}$ is the number of spikes (by all neurons or during all the trials) generated during a given simulation run. For some calculations it is convenient to pool the spike times of all neurons together into one set, $\left\{t_{1}, \ldots, t_{\nu}, \ldots, t_{N_{s}}\right\}$, ordered from low to high values. The ordered set is indexed by $\nu$, where $\nu=1$ is the earliest spike and $\nu=N_{s}$ is the latest. Most of the statistical quantities determined from the simulations were spike based; that is, for each measurement $y_{\nu}$ there is an associated spike time $t_{\nu}$. For instance, $y_{\nu}$ could be an interspike interval and $t_{\nu}$ could be the first spike time of the interval. The specific choices for $y$ are given below. In some cases the simulation runs were divided into three intervals: (i) $0<t<750 \mathrm{~ms}$, when the first ensemble is in a chaotic state; (ii) $750 \mathrm{~ms}<t<1500 \mathrm{~ms}$, when the first ensemble is phase locked, and (iii) $1500 \mathrm{~ms}$ $<t<2250 \mathrm{~ms}$, when the first ensemble has returned to a 
chaotic state. Averages of $y$ were determined for each of these intervals, using all the $y_{\nu}$ that had a $t_{\nu}$ in the interval under consideration. Since the $t_{\nu}$ are ordered, this is a contiguous set, $\nu_{b} \leqslant \nu \leqslant \nu_{e}$ ( $b$ stands for begin and $e$ stands for end).

$$
\langle y\rangle=\frac{1}{\nu_{e}+1-\nu_{b}} \sum_{\nu=\nu_{b}}^{\nu_{e}} y_{\nu}, \quad\langle t\rangle=\frac{1}{\nu_{e}+1-\nu_{b}} \sum_{\nu=\nu_{b}}^{\nu_{e}} t_{\nu} .
$$

We also determined the time-resolved average using a sliding window of length $T_{a v}$ that was translated along the time axis with increments equal to $T_{\text {incr }}$. The position of the sliding window was indicated by the index $k$. The resulting averages $\langle y\rangle_{k}$ and $\langle t\rangle_{k}$ are over all $\nu$ values with $(k-1) T_{\text {incr }} \leqslant t_{\nu}$ $<(k-1) T_{\mathrm{incr}}+T_{a v} . T_{a v}$ and $T_{\mathrm{incr}}$ are expressed in terms of $n_{a v}$ and $n_{\text {incr }}$ using the relations $T_{a v}=T_{\max } / n_{a v}$ and $T_{\text {incr }}$ $=\left(T_{\max }-T_{a v}\right) / n_{\text {incr }}$. We used $n_{a v}=25, n_{\text {incr }}=75$, and $T_{\max }$ $=2500 \mathrm{~ms}$ [the last $250 \mathrm{~ms}$ of the simulation were not included in interval (iii) in order to make its length equal to that of interval (ii)].

Standard spike train statistics. The mean firing rate $f$ is defined as the number of spikes divided by the duration of the measurement interval. The mean firing rate is determined for each time interval [(i)-(iii)]. The neuron was driven by an oscillatory current with period $T=1 / f_{d}=25 \mathrm{~ms}$. Since the firing rate of the neuron was less than $40 \mathrm{~Hz}$, there would not be more than one spike per cycle [the $n$th cycle was the time interval between $(n-1) T$ and $n T$ ]. The jitter $\sigma$ is defined as the standard deviation across trials of the spike times on a given cycle, averaged across all cycles during the time interval of interest.

Binned spike train statistics. A binned representation $X_{n}(t)$ of the spike train of the $n$th neuron is obtained by setting $X_{n}(t)$ equal to the number of spikes between $t$ $-\Delta t / 2$ and $t+\Delta t / 2$. For the model neuron used here the relative refractory period is larger than $2 \mathrm{~ms}$. Hence, for small binwidth $\Delta t \leqslant 2 \mathrm{~ms}$, there cannot be more than one spike per bin. The spike time histogram, STH $=(1000 \Delta t / N) X(t)$, is proportional to the sum of $X_{n}$ over all neurons, $X=\sum_{n=1}^{N} X_{n}$ (since $\Delta t$ is expressed in milliseconds, the factor 1000 assures that STH is in hertz).

Interspike-distance reliability and synchrony measures. The $C_{V}^{P}$ measure is based on the idea that during reliable (synchronous) states the minimum distance between spikes of different neurons is reduced compared with unreliable (asynchronous) states. The interspike interval of the combined set of ensemble spikes is $\tau_{\nu}=t_{\nu+1}-t_{\nu}$. Note that these interspike intervals are between different neurons. The coefficient of variation is

$$
C_{V}^{P}=\frac{\sqrt{\left\langle\tau_{\nu}^{2}\right\rangle_{\nu}-\left\langle\tau_{\nu}\right\rangle_{\nu}^{2}}}{\left\langle\tau_{\nu}\right\rangle_{\nu}}
$$

Here $P$ stands for population and $\langle\cdot\rangle_{\nu}$ is the average over all intervals. The interval $\tau_{\nu}$ can be identified with three times $t_{\nu}, t_{\nu+1}$, and the mean $\left(t_{\nu}+t_{\nu+1}\right) / 2$. For the sliding-window average, $C_{V}^{P}(t)$, the latter was used. $C_{V}^{P}$ depends only on the

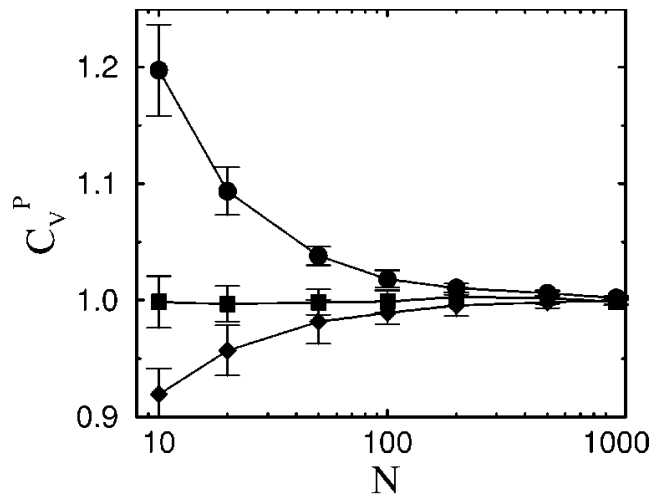

FIG. 2. The $C_{V}^{P}$ of an asynchronous ensemble of $N$ neurons converges to 1 . For this example, each neuron produced a random spike train with $\mathrm{CV} \approx 1.52$ (circles), $\mathrm{CV}=1$ (Poisson spike train, squares), and $\mathrm{CV} \approx 0.707$ (diamonds). The construction of the spike trains is described in the text. Error bars are the standard deviation across ten different realizations of the ensemble of spike trains.

aggregate set of spike times and it is not sensitive to which neuron produced a given spike. Hence, it can not be used to distinguish, for instance, a Poisson process from a Gamma process with an order $\nu>1$ [33].

For an asynchronous ensemble each neuron fired independently at a constant rate. The spike time histogram is therefore approximately constant as a function of time. When each neuron produces a homogeneous Poisson spike train, the CV (coefficient of variation, or the standard deviation of the single neuron's interspike intervals divided by the mean) will be equal to one. The sum of independent Poisson processes is also a Poisson process. The coefficient of variation $C_{V}^{P}$ of the aggregate spike train is therefore also one. The variance in the $C_{V}^{P}$ value across different realizations of the ensemble only depends on the number of spikes $N_{s}$ in the aggregate spike train, not on the number of neurons $N$ in the ensemble. (Numerically we found that for $N_{s}=10$ spikes, $C_{V}^{P}=0.92 \pm 0.24$, for $N_{s}=100, C_{V}^{P}=0.99 \pm 0.10$, and for $N_{s}$ $=1000, C_{V}^{P}=1.00 \pm 0.03$, errors were the standard deviation across 1000 realizations.) When the neuron produced random spike trains that were not Poisson and had CVs different from one, the value of $C_{V}^{P}$ did depend on $N$. We conjecture that for large enough ensembles $C_{V}^{P} \approx 1$ in the asynchronous state. We performed simulations to test this conjecture and determined at what $N$ value $C_{V}^{P}$ approached unity (Fig. 2). Random spike trains with a CV of less than one were generated from Poisson spike trains by removing every other spike. The expected value of the CV was $1 / \sqrt{2} \approx 0.707$ and the mean interspike interval was $20 \mathrm{~ms}$. Random spike trains with a CV larger than one were generated using an interspike interval distribution that was the sum of two exponential distributions: distribution 1 had a mean of $1 \mathrm{~ms}$ and distribution 2 had a mean of $10 \mathrm{~ms}$. This type of bimodal distribution is representative of a neuron firing doublets. For a given spike train the even ISIs came from distribution 1 and the odd ISIs from distribution 2. In that way we obtain a sequence consisting of a repeating motif of a long interval followed by a short interval. The actual spike times are given by the cumulative sum over all the ISIs. The CV of the 
resulting spike train was $\approx 1.523$. For each spike train, a transient of $100 \mathrm{~ms}$ was discarded and the remaining 1500 ms were used for analysis. For an ensemble of $N=10$ spike trains with $\mathrm{CV}$ values different from one, the $C_{V}^{P}$ was significantly different from 1 , but, for $N=1000$, the ensembles had $C_{V}^{P}$ values close to one (Fig. 2). Based on these numerical results we expect that for sets of a few hundred spike trains with a constant rate and a CV different from one, the $C_{V}^{P}$ for the asynchronous state will be close to unity.

For a perfectly synchronous (reliable) ensemble with $N$ neurons oscillating with period $T$, the set $\left\{\tau_{\nu}\right\}$ consists of $N_{s} / N-1$ intervals equal to $T$ and $N_{s}-N_{s} / N$ short intervals between spikes that are part of the same event. We evaluate $C_{V}^{P}$ in the limit that the length $\Delta$ of these short intervals goes to zero. Hence, to first order in $\Delta$,

$$
C_{V}^{P}=\sqrt{\alpha}[1-(\alpha / T+T / \alpha) \beta \Delta],
$$

with $\alpha=\left(N_{s}-1\right) /\left(N_{s} / N-1\right)$ and $\beta=\left(N_{s} / N\right)(N-1) /\left(N_{s}\right.$ $-1)$. For large $N_{s}, C_{V}^{P}$ reduces to $\sqrt{N}$. Thus, $\left(C_{V}^{P}-1\right) / \sqrt{N}$ is a measure for synchrony that is normalized between 0 and 1 . Since $N$ is constant, we will use $C_{V}^{P}$ without this normalization. $C_{V}^{P}$ is sensitive to the precision of the ensemble discharge as well as to the degree of coincidence.

The nearest-neighbor distance of spike $t_{n i}$ from the spikes of neuron $m$ is

$$
\Delta_{m n i}=\min _{j}\left|t_{n i}-t_{m j}\right|
$$

the minimum is taken over all spike times $t_{m j}$ of neuron $m$. There are $N_{s}$ different spikes and for each spike there are $N-1$ different nearest-neighbor distances to the other neurons in the ensemble. Hence, there are $N_{p}=(N-1) N_{s}$ nearest-neighbor distances, referred to as pairs. Note that each pair is counted twice, $\Delta_{m n i}=\Delta_{n m j}$, because it is associated with $t_{n i}$ as well as with $t_{m j}$. The coincidence factor $\kappa$ is defined as the number of $\Delta_{m n i}<P$, divided by the maximum number of coincidences $N_{c}$, here $P$ is a preset precision, typically equal to $2 \mathrm{~ms}$. The number of $\Delta_{m n i}<P$ cannot exceed $N_{p}$, hence taking $N_{c}=N_{p}=(N-1) N_{s}$ would yield a number between 0 and 1 (note that $N_{s}$ is now the total number of spikes in the interval under consideration). However, when the minimum single-neuron interspike interval is larger than $P$ for all neurons, the number of coincidences in a pair of neurons $(n, m)$ cannot be larger than the minimum of the number of spikes produced by neurons $n$ and $m$, formally equal to $\min \left(\Sigma_{t} X_{n}(t), \Sigma_{t} X_{m}(t)\right)$. Hence, the normalization should be

$$
N_{c}=\sum_{n \neq m} \min \left(\sum_{t} X_{n}(t), \sum_{t} X_{m}(t)\right) .
$$

The sliding-window average $\kappa(t)$ was calculated for each window as the number of coincidence $\Delta_{m n i}<P$ divided by $N_{c}$. The $\kappa$ defined here is adapted from a measure described in Refs. $[17,28]$. It was modified in order to yield correct results for ensembles consisting of neurons with different mean firing rates [34] so that it could resolve fast temporal modulations in the degree of coincidence.

An estimate for the dispersion of the spike times in an event can be obtained from $\Delta_{m n i}$. For a given spike time $t_{n i}$ we take the mean of the $N_{\Delta}$ lowest values of $\Delta_{m n i}$. This value is averaged across all $t_{n i}$ in the averaging time interval of length $T_{a v}$ to obtain $\Delta(t)$. When $N_{\Delta}$ is somewhat smaller than the mean number of neurons firing on each cycle, $\Delta(t)$ is proportional to the dispersion in the spike times.

Spike metrics. The distance between spike trains was quantified using the metrics introduced by Victor and Purpura [35]. The metric based on the spike times was $D^{t}(q)$, and the one based on the interspike intervals was $D^{\tau}(q)$. The distance between two spike trains was the minimum cost to transform one spike train into the other by moving or deleting existing spike times (interspike intervals) or adding spike times (interspike intervals). The cost of adding or deleting a spike (interval) was one and the cost was $q$ to move a spike over $1 \mathrm{~ms}$ (increase or decrease an interval by $1 \mathrm{~ms}$ (Ref. [68])).

Our goal was to determine the statistics of the distance between the output spike train generated in response to a set of attractor spike trains and the output spike train generated in response to surrogate spike trains. The spike trains produced by the neurons of the first ensemble did not satisfy Poisson statistics [18], to indicate this we refer to them as "attractor spike trains" or sometimes as "original spike trains." Surrogate spike trains were obtained by randomly distributing spike times across the different neurons in the first ensemble. After this procedure short segments in the spike train have Poisson statistics [18,36]. We implemented this in MATLAB using two arrays: one containing the spike times, and the other containing the index of the neuron that produced the spike. The elements of the array containing the neuron index were then randomly permuted using RANDPERM. Ten sets of attractor spike trains and ten sets of surrogate spike trains were generated, and the conductance wave forms corresponding to each of these sets were injected into the model neuron on 40 trials. This yielded 800 output spike trains. The spike trains were labeled by the input-spike-trainset indices $\nu$ and $\mu$ and the trial indices $n$ and $m$. $D_{\nu n, \mu m}$ was the distance between the output spike trains labeled by $\nu n$ and $\mu m$, respectively. $D_{\nu \mu}$ was the distance between the spike trains generated in response to set $\mu$ and those generated in response to set $\nu$ averaged over all pairs $(n, m)$ of trials $n=1, \ldots, 40$ belonging to set $\nu$ and $m=1, \ldots, 40$ belonging to set $\mu$. For the diagonal entries $D_{\nu \nu}$, the average was over all pairs of different spike trains $(n \neq m)$. The matrix elements were divided into three groups: distances between output spike trains generated by $(a a)$ two sets of attractor spike trains $(\nu=1, \ldots, 10, \mu=1, \ldots, 10),(p p)$ two sets of surrogate spike trains $(\nu=11, \ldots, 20, \mu=11, \ldots, 20)$, and $(a p)$ between a set of attractor spike trains and a set of surrogate spike trains $(\nu=1, \ldots, 10, \mu=11, \ldots, 20)$, where " $a$ " stands for attractor and " $p$ " stands for Poisson (surrogate). The distance was also averaged over all elements of a given group, yielding $D_{a a}^{\tau}(q), D_{p p}^{\tau}(q), D_{a p}^{\tau}(q)$, $D_{a a}^{t}(q), D_{p p}^{t}(q)$, and $D_{a p}^{t}(q)$. 

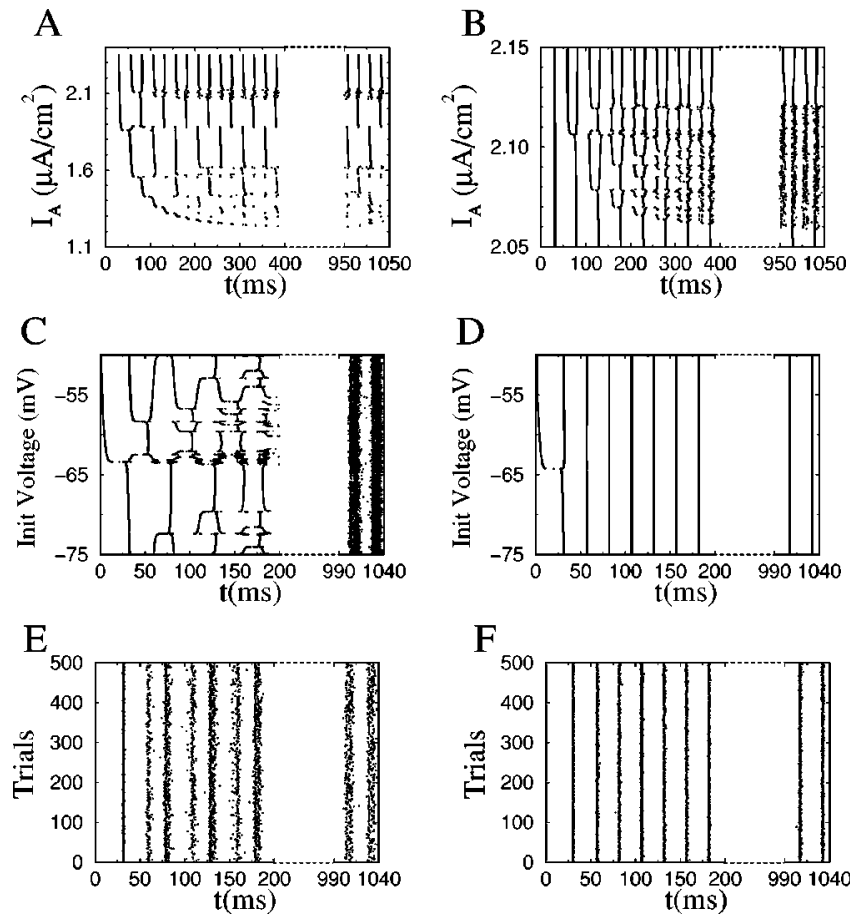

FIG. 3. Dependence of the reliability on the stimulus amplitude $I_{A}$. In each panel we show spike times as ticks in a rastergram: the $x$ coordinate is the spike time and the $y$ coordinate is either the amplitude $I_{A}$ of the driving force (A,B), or the value of the voltage at the start of the simulation (C,D), or else the trial index (E,F). For all simulations, $I_{0}=0 \mu \mathrm{A} / \mathrm{cm}^{2}$ with $(\mathrm{A}, \mathrm{B}) D=0$ and $\left(D, I_{A}\right)=(\mathrm{C})$ $(0,2.08)$, (D) $(0,2.3),(\mathrm{E})(0.004,2.08)$, and (F) $(0.004,2.3)$. $D$ was expressed in $\mathrm{mV}^{2} / \mathrm{ms}$ and $I_{A}$ in $\mu \mathrm{A} / \mathrm{cm}^{2}$.

The diagonal elements $D_{\nu \nu}^{t}\left(D_{\nu \nu}^{\tau}\right)$ represented the variability in the spike trains elicited by the same input across different trials. We determined the mean $D_{a}^{t}\left(D_{a}^{\tau}\right)$ of $D_{\nu \nu}^{t}\left(D_{\nu \nu}^{\tau}\right)$ across all sets of attractor spike trains $(\nu=1, \ldots, 10)$ and the mean $D_{p}^{t}\left(D_{p}^{\tau}\right)$ across all sets of surrogate spike trains $(\nu$ $=11, \ldots, 20)$. The distance $D_{\nu \mu}^{t}\left(D_{\nu \mu}^{\tau}\right)$ was normalized by $D_{a}^{t}\left(D_{a}^{\tau}\right)$.

\section{RESULTS}

\section{A. Dependence of neural response reliability on the stimulus amplitude}

We examined the spike trains produced by the model neuron when it was driven with a sinusoidal current. The driving frequency $f_{d}$ was $40 \mathrm{~Hz}$ and the amplitude $I_{A}$ was varied. The mean depolarizing current $I_{0}$ was zero, hence, the neuron was in an excitable state and would not spike in the absence of the oscillating current. There was no intrinsic noise, $D=0$. For each value of $I_{A}$, the simulation was started with an initial voltage $V=-75 \mathrm{mV}$ and the spike times during a $1000 \mathrm{~ms}$ interval were recorded. The spike times were plotted as ticks in a rastergram, the $y$ coordinate of the tick was $I_{A}$ and the $x$ coordinate was the spike time [Fig. 3(A)]. The neuron started spiking when the drive amplitude $I_{A}$ exceeded $1.22 \mu \mathrm{A} / \mathrm{cm}^{2}$. The neuron became phase locked for specific contiguous ranges of the driving current
$I_{A}$. These locking regimes were visible in the rastergram as a sequence of straight lines on which the spike time varied only weakly with $I_{A}$. During locking the firing rate of the neuron was constant and equal to a fraction, $(n / m) f_{d}$, of the drive frequency. For $1.63 \mu \mathrm{A} / \mathrm{cm}^{2} \leqslant I_{A} \leqslant 2.055 \mu \mathrm{A} / \mathrm{cm}^{2}$, the neuron produced one spike $(n=1)$ on every two cycles $(m$ $=2$ ). For the initial condition used here, the neuron spiked on the even cycles for $1.63 \mu \mathrm{A} / \mathrm{cm}^{2} \leqslant I_{A} \leqslant 1.85 \mu \mathrm{A} / \mathrm{cm}^{2}$, and on the odd cycles for $1.85 \mu \mathrm{A} / \mathrm{cm}^{2}<I_{A}$ $\leqslant 2.055 \mu \mathrm{A} / \mathrm{cm}^{2}$ [the $n$th cycle was the time interval between $(n-1) T$ and $n T$, where $\left.T=1 / f_{d}=25 \mathrm{~ms}\right]$. The neuron produced one spike per cycle for $I_{A} \geqslant 2.13 \mu \mathrm{A} / \mathrm{cm}^{2}$ (the phase-locking range extended to at least $I_{A}=7.00 \mu \mathrm{A} / \mathrm{cm}^{2}$, but this value was outside the physiological range). In between the locking zones there were zones where the spike time varied nonmonotonically with the driving current and where the firing rate was not constant. We further investigated the current range between $I_{A}=2.06 \mu \mathrm{A} / \mathrm{cm}^{2}$ and $2.12 \mu \mathrm{A} / \mathrm{cm}^{2}$ [Fig. 3(B)]. The first spike time was on the second cycle for all values of $I_{A}$. However, the second spike was on the third cycle for some values of $I_{A}$, and on the fourth cycle for others. With each consecutive spike, the range of contiguous current values that had the same spike times up to the present cycle splits up in two groups with different spike times. After about $1000 \mathrm{~ms}$ the spike raster did not show much structure. The lack of structure was twofold: the cycle on which a spike was obtained for a given current looked stochastic, but the actual phase of the spike within the cycle also had a larger jitter. The phase of a spike depended, due to the pronounced afterhyperpolarization, on the sequence of interspike intervals that led to that particular spike.

We compared the dependence on initial conditions for a phase-locked solution, $I_{A}=2.30 \mu \mathrm{A} / \mathrm{cm}^{2}$, with that for a nonlocked solution, $I_{A}=2.08 \mu \mathrm{A} / \mathrm{cm}^{2}$. We took a uniformly spaced grid of initial voltages between $V=-75 \mathrm{mV}$ and $-50 \mathrm{mV}$ with 5000 grid points for $I_{A}=2.08 \mu \mathrm{A} / \mathrm{cm}^{2}$ and 500 grid points for $I_{A}=2.30 \mu \mathrm{A} / \mathrm{cm}^{2}$. For $I_{A}=2.08 \mu \mathrm{A} / \mathrm{cm}^{2}$ [Fig. 3(C)], the rasters looked similar to those shown in Fig. 3(B) the first spike of the trial either occurred on the first or second cycle. But part of the initial conditions that led to a first spike on the first cycle led to a spike on the second cycle, whereas the rest led to a spike on the third cycle. Hence, the contiguous set of initial conditions that led to the same sequence of spikes again splits up into smaller sets with each consecutive cycle. After $1000 \mathrm{~ms}$, these contiguous sets have become smaller than the resolution of the simulation grid and the dependence of spike time on the initial condition looks random. This sensitive dependence on initial conditions is indicative of a chaotic system [37]. Although we did not quantify the degree of chaos using the positivity of the Lyapunov exponent, we will still refer to this state as chaotic in the remainder. For $I_{A}=2.30 \mu \mathrm{A} / \mathrm{cm}^{2}$, the first spike either occurred on the first or second cycle. However, starting from the third cycle all the spike times occurred at the same time and the spikes remained lined up for the duration of the trial. Hence, there was a stark contrast between the dependence on initial conditions for these two cases. In the former case, the spike trains became more dis- 
similar as time went on, whereas in the latter case the spike trains converged within a few cycles to the same spike train.

For integrate-and-fire neurons robustness against different initial conditions was related to the robustness against intrinsic noise [38]. We performed 500 simulations starting from the same initial condition $V=-75 \mathrm{mV}$, but each with a different random realization of the intrinsic noise [Fig. 3(E) and 3(F)]. The variance of the intrinsic noise was $D$ $=0.004 \mathrm{mV}^{2} / \mathrm{ms}$. For $I_{A}=2.08 \mu \mathrm{A} / \mathrm{cm}^{2}[$ Fig. 3(E)], the jitter in the first spike time was small, $\sigma=0.45 \mathrm{~ms}$, but it increased with time and saturated at $\sigma=3.06 \mathrm{~ms}$. The firing rate of the neuron was $f=29.80<40 \mathrm{~Hz}$ and it skipped cycles. The skipping of cycles was random across trials and the reliability was reduced. For $I_{A}=2.3 \mu \mathrm{A} / \mathrm{cm}^{2}$ [Fig. 3(F)], the jitter in the first spike time was $\sigma=0.24 \mathrm{~ms}$ and saturated to $\sigma=0.56 \mathrm{~ms}$ at the end of the trial. The neurons spiked on each cycle during each trial, indicating perfect reliability.

The above results show that a modest change in the amplitude of the driving force can result in large changes in the reliability and precision of the spike trains across trials. In the following we interpret the response across multiple trials as the response of multiple neurons across one trial, and determine how it modulates the response of a receiving ensemble.

\section{B. Modulation of firing rate by the reliability of input spike trains}

The dynamics of a neural circuit consisting of two ensembles was studied. The first ensemble consisting of $N$ $=500$ neurons was driven by a current oscillating at $40 \mathrm{~Hz}$. The amplitude was transiently increased from $I_{A}$ $=2.08 \mu \mathrm{A} / \mathrm{cm}^{2}$ to $2.3 \mu \mathrm{A} / \mathrm{cm}^{2}$ between $t=750 \mathrm{~ms}$ and $1500 \mathrm{~ms}$ [Fig. 4(Aa)]. The change of amplitude represented a transient increase of the power in the gamma-frequency range that was observed in local field potentials [24] and electroencephalographic recordings [21,22]. During the transient increase in amplitude, the firing rate, reliability, and precision were increased (Table I). For each neuron in the second ensemble, a different set of $N_{\text {con }}=100$ spike trains was randomly selected from the $N=500$ neurons (trials) in the ensemble and was convolved with the shape of the excitatory postsynaptic conductance (EPSC) to obtain the synaptic conductance. The unitary EPSC was exponential with amplitude $\delta g=3 \times 10^{-4} \mathrm{mS} / \mathrm{cm}^{2}$. We first studied the case without STD. The amplitude was selected so that the excitatory postsynaptic potentials (EPSPs) summed across input neurons had a magnitude between 3 and $6 \mathrm{mV}$. The synaptic conductance wave form was injected in each neuron of the second ensemble with a white noise current (the variance was $D=0.04 \mathrm{mV}^{2} / \mathrm{ms}$ ) that was independent across the ensemble of neurons. Note that $D$ is an order of magnitude larger compared with that injected into the first ensemble (see the Discussion). During the transient increase in gamma power the firing rate of the second ensemble was strongly increased [Fig. 4(Ae)]. The precision and reliability also increased (Table I).

The modulations in reliability and precision were measured using three statistics, $C_{V}^{P}, \kappa$, and $\Delta$ [Figs. 4(B) and
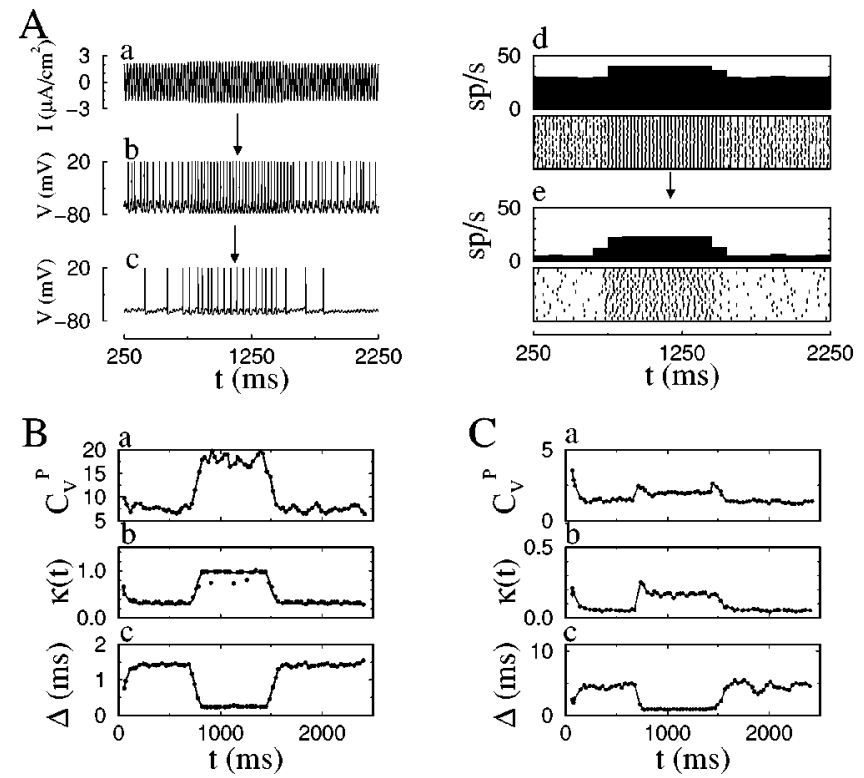

FIG. 4. Transient increases in reliability and precision boosted the firing rate in downstream cortical neurons. (A) (a) The sinusoidal driving current and voltage traces for (b) a neuron in the first ensemble and (c) one in the second ensemble. (d,e) In each panel we plot (top) the spike time histogram and (bottom) the rastergram of 20 neurons of (d) the first ensemble and (e) the second ensemble. (B,C) In each panel, we show (a) the population coefficient of variation $C_{V}^{P}$, (b) sliding average of the degree of coincidence $\kappa(t)$, and (c) the sliding average of the jitter estimate $\Delta(t)$, for the spike trains of $\left(\mathrm{B}, N_{\Delta}=200\right)$ the first ensemble and $(\mathrm{C}$, $\left.N_{\Delta}=50\right)$ the second ensemble. Parameters for the first ensemble were $D=0.004 \mathrm{mV}^{2} / \mathrm{ms}$ and $I_{0}=0$. During the time interval $t$ $=750-1500 \mathrm{~ms}, \quad I_{A}$ was increased from $2.08 \mu \mathrm{A} / \mathrm{cm}^{2}$ to $2.3 \mu \mathrm{A} / \mathrm{cm}^{2}$. The second ensemble was driven by the spike trains of the first ensemble; each spike resulted in a unitary EPSC with an amplitude $3 \times 10^{-4} \mathrm{mS} / \mathrm{cm}^{2}$ and a decay constant of $3 \mathrm{~ms}$. $I_{0}$ was equal to $-0.3 \mu \mathrm{A} / \mathrm{cm}^{2}$.

4(C)]. $C_{V}^{P}$ corresponds to a mix of precision and reliability, it is the coefficient of variation of the interspike intervals of the aggregate spike trains. Note that a high coefficent of variation of the interspike intervals of a single neuron implies high variability. In contrast, a high $C_{V}^{P}$ implies high reliability, that is, less variability in spike trains across trials. $\kappa$ is the sliding average of the number of coincidences (pairs of spike times from different neurons that differ by less than $2 \mathrm{~ms}$ ) normalized by the number of possible coincidences and $\Delta$ is a time-resolved measure proportional to the jitter. Modulations in the precision correspond to the inverse of $\Delta$, whereas modulations in the reliability are directly related to $\kappa$. In both ensembles there was a robust increase in $C_{V}^{P}$ and $\kappa$, and a robust decrease in $\Delta$ during the transient increase in gamma power [interval (ii) in Table I].

We also performed simulations where each neuron in the second ensemble received inputs from the same set of $N_{\text {con }}$ $=100$ spike trains (not shown). Hence, the same synaptic conductance wave form was injected in each neuron together with a white noise current that was independent across the ensemble of neurons. The results obtained in that way were 
TABLE I. Spiking statistics for ensembles 1 and 2. The firing rate $f, C_{V}^{P}$, degree of coincidence $\kappa$, and jitter $\Delta$ were calculated during interval (ii) (750-1500 ms) and interval (iii) (1500-2250 ms) as described in Sec. II. We calculated $\Delta$ using $N_{\Delta}=200$ neighbors for the first ensemble and $N_{\Delta}=50$ for the second ensemble. Results labeled by the asterisk are obtained by driving each neuron in the second ensemble by the same 100 neurons of the first ensemble.

\begin{tabular}{lccccc}
\hline \hline & Time interval & $f(\mathrm{~Hz})$ & $C_{V}^{P}$ & $\kappa$ & $\Delta(\mathrm{ms})$ \\
\hline Ensemble 1 & (ii) & 39.96 & 17.15 & 0.96 & 0.26 \\
Ensemble 1 & (iii) & 29.80 & 7.58 & 0.33 & 1.38 \\
Ensemble 1, Poisson resampled & (ii) & 39.96 & 17.15 & 0.68 & 0.30 \\
Ensemble 1, Poisson resampled & (iii) & 29.80 & 7.58 & 0.30 & 1.68 \\
Ensemble 2 $(*)$, no STD & (ii) & 22.16 & 2.12 & 0.16 & 0.98 \\
Ensemble 2 $(*)$, no STD & (iii) & 5.83 & 1.82 & 0.06 & 3.70 \\
Ensemble 2, no STD & (ii) & 22.23 & 2.05 & 0.16 & 0.99 \\
Ensemble 2, no STD & (iii) & 5.53 & 1.48 & 0.05 & 3.99 \\
Ensemble 2 (*), STD & (ii) & 15.56 & 2.01 & 0.11 & 1.34 \\
Ensemble 2 $*)$, STD & (iii) & 8.63 & 1.68 & 0.06 & 2.53 \\
Ensemble 2 (*), Poisson input, STD & (ii) & 13.25 & 2.27 & 0.13 & 1.56 \\
Ensemble 2 $*$ ), Poisson input, STD & (iii) & 7.67 & 3.09 & 0.08 & 2.60 \\
\hline \hline
\end{tabular}

similar to those shown Fig. 4 (see Table I for a comparison).

The increase in firing rate of the second ensemble due to reliability and precision modulations induced by the transient increase in gamma power depended on the value of the constant driving current $I_{0}$ [Fig. 5(A) and 5(B)]. $I_{0}$ was $-0.3 \mu \mathrm{A} / \mathrm{cm}^{2}$ for the data shown in Fig. 4. For more negative values of $I_{0}$ the neuron would hardly spike during time intervals (i) and (iii), hence the relative increase in firing rate during time interval (ii) was even larger. For more positive values of $I_{0}$, the increase in firing rate was reduced. For $I_{0}$ between $0.1 \mu \mathrm{A} / \mathrm{cm}^{2}$ and $0.2 \mu \mathrm{A} / \mathrm{cm}^{2}$, both firing rates $f_{1}$ [during interval (ii)] and $f_{2}$ [during interval (iii)] were equal to the driving frequency $f_{d}$ of the sinusoidal current (which
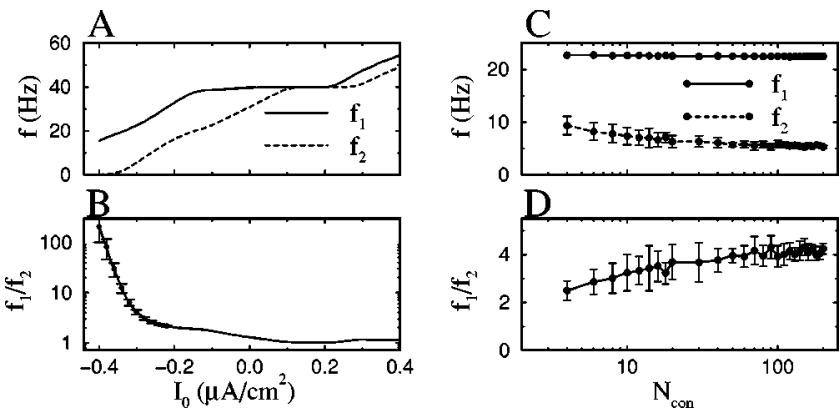

FIG. 5. Firing rate modulation by input reliability depends on the depolarizing current $I_{0}$ and the number of inputs $N_{\text {con }}$ (A,C) Firing rate $f_{1}$ during the time interval 750-1500 ms and $f_{2}$ during 1500-2250 ms plotted vs (A) $I_{0}$ and (C) $N_{\text {con }}$. (B,D) The ratio $f_{1} / f_{2}$ vs (B) $I_{0}$ and (D) $N_{\text {con }}$. Parameters were the same as in Fig. 4 except that $(\mathrm{A}, \mathrm{B}) I_{0}$ for the neurons in the second ensemble was varied and $(\mathrm{C}, \mathrm{D})$ the number of input neurons was varied and the unitary conductance was rescaled according to $\delta g$ $=\left(3 \times 10^{-2}\right) / N_{\text {con }} \mathrm{mS} / \mathrm{cm}^{2}$. The error bars were the standard deviation across ten different random realizations of the $N_{\text {con }}$ inputs to the neuron. The error in $f_{1}$ and $f_{2}$ in (A) and $f_{1}$ in (C) was smaller than the symbol size. was only injected in neurons of the first ensemble). Concomitantly, the synchrony of the second ensemble reached its highest value, $C_{V}^{P} \approx 8.6$, on the step of constant firing rate. However, the $C_{V}^{P}$ did not vary strongly with current on the step. The synchrony decreased again for higher values of $I_{0}$. The range of driving currents $I_{0}$ for which the firing rate was sensitive to the reliability and precision of the input increased with the size of the unitary excitatory conductance.

The firing rate modulation could also potentially depend on the number of inputs that the neurons in the second ensemble received. To investigate this issue we varied $N_{\text {con }}$ and rescaled the unitary conductance $\delta g$ so that the mean synaptic conductance remained constant. Hence, $\delta g$ was much larger for a small value of $N_{\text {con }}$. The firing rate $f_{1}$ did not depend on the value $N_{\text {con }}$ [Fig. 5(C)]. In contrast, $f_{2}$ decreased with increasing $N_{\text {con }}$ [Fig. 5(C)], as a result the boost in firing rate $f_{1} / f_{2}$ also increased with $N_{\text {con }}$ [Fig. 5(D)]. The changes in $f_{2}$ and $f_{1} / f_{2}$ saturated for $N_{\text {con }}>100$. These results indicate that there is a broad range of $N_{\text {con }}$ values for which precision and reliability of the inputs modulate the firing rate.

\section{Effect of non-Poisson statistics of input spike trains}

In the preceding section there was no STD of the synapses: Each presynaptic spike yielded the same unitary conductance amplitude independent of the timing of the previous spikes produced by the presynaptic neuron. Hence, the synaptic conductance wave form only depends on the distribution of presynaptic spike times - the spike time histogram. The spike trains produced by neurons driven by fluctuating currents in the presence of weak intrinsic noise do not form a renewal process because the interspike intervals are correlated [18]. Hence, they do not satisfy Poisson statistics. We refer to these spike trains as attractor spike trains or original spike trains. Surrogate spike trains were obtained by ran- 

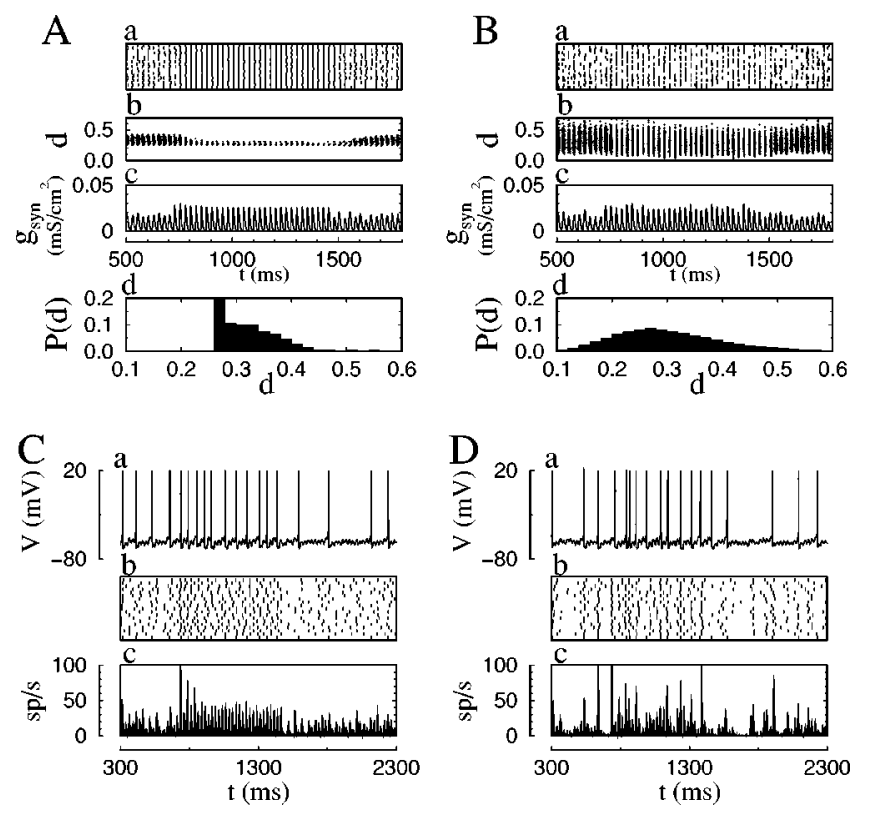

FIG. 6. Downstream responses are sensitive to spike patterns. $(A, B)$ In each panel, we show (a) 20 spike trains from the first ensemble in a rastergram, (b) a scatterplot of the depression variable $d(t)$, where the $x$ coordinate is the arrival time of the EPSC and the $y$ coordinate is the value of $d(t)$, (c) synaptic conductance wave form, and (d) histogram of the $d$ values for all EPSCs. (C,D) In each panel we show (a) voltage trace of one neuron, (b) 20 spike trains, and (c) spike time histogram for the second ensemble. Data in $(\mathrm{A}, \mathrm{C})$ are obtained using the original (attractor) spike trains of the first ensemble; the data in (B,D) are for Poisson surrogate spike trains. Parameters were as in Fig. 4 except that there was short term depression and the unitary conductance was increased to $\delta g$ $=1 \times 10^{-3} \mathrm{mS} / \mathrm{cm}^{2}$.

domly distributing spike times across the different neurons in the first ensemble. After this procedure the spike trains satisfy Poisson statistics. The original spike trains as well as the surrogate spike trains have, by definition, the same spike time histogram. The synaptic conductance wave form and the postsynaptic response of the second ensemble to these spike trains will be identical. However, when the synapses have STD the amplitude of the unitary synaptic conductance depends on the value of preceding interspike intervals (see the Methods). The synaptic conductance wave form will be different for the original spike trains compared with that for the surrogate spike trains. The question is to what extent these differences affect the firing rate and spike timing of the second ensemble. To address this issue we compared the response of the second ensemble to the attractor spike trains of the first ensemble [Figs. 6(A) and 6(C)] with the response to surrogate spike trains with Poisson statistics [Fig. 6(B) and 6(D)]. For ease of comparison, each neuron in the second ensemble received inputs from the same set of $N_{\text {con }}=100$ neurons. The corresponding rastergrams look different [Figs. 6(Aa) and 6(Ba)]. In the surrogate spike train there could be $0,1,2$ or more spikes on a cycle during interval (ii), whereas in the original spike train there usually was only one. This led to gaps in the rastergram shown in Fig. 6(Ba) during interval (ii), which were not present in Fig. 6(Aa). Hence, it seems as if the firing rate of the Poisson-resampled first ensemble had decreased, but in both cases there were exactly the same number of spikes. The resampling also resulted in a broader distribution of $d(t)$ values. $d(t)$ is shown as a function of time in Figs. 6(Ab) and 6(Bb) and its distribution is shown in Figs. 6(Ad) and 6(Bd). The synaptic drive injected into neurons of the second ensemble was more variable for Poisson statistics [Fig. 6(Bc)] compared with that for the original spike trains [Fig. 6(Ac)]. These differences in the synaptic conductance waveforms led to different spike trains [Figs. 6(Ca) and 6(Da), respectively]. This difference persisted across trials [Fig. 6(Cb) and 6(Db), respectively] and was especially salient in the spike time histogram. The height of the peaks for Poisson statistics [Fig. 6(Dc)] was more variable than those for the original input (Fig. 6Cc). Despite these differences the firing rate during interval (ii) was still higher than that during interval (iii) in response to both types of input spike trains.

\section{Statistical analysis of the difference between attractor and surrogate responses}

Figure 6 shows that the spike train response to an input consisting of attractor spike trains (attractor response for short) is different from the response to surrogate spike trains with Poisson statistics (surrogate response for short). The difference between the responses depended on the specific random realization of the set of input spike trains, that is, which of the $N_{\text {con }}=100$ spike trains were picked randomly among the $N=500$ neurons in the first ensemble. To determine whether the differences observed in Fig. 6 were statistically significant, we compared the responses across 20 different random realizations of the set of input spike trains, comprised of ten sets of attractor spike trains and ten sets of surrogate spike trains. The pairwise distance between spike trains was quantified using the metrics introduced by Victor and Purpura [35]. Spike trains are more dissimilar when the distance between them is larger. Our implementation is described in the Methods section. Briefly, the metric was based on the set of interspike intervals $D^{\tau}(q)$ or spike times $D^{t}(q)$ and depended on a parameter $q$. The distances were expressed as the matrix $D_{\nu \mu}^{\tau}\left(D_{\nu \mu}^{t}\right)$. The diagonal elements are the mean distance between spike trains generated across multiple trials in response to the same set of input spike trains. Hence, $D_{\nu \nu}$ is a measure for the variability of the response due to intrinsic noise sources. The parameter $q$ weighs the relative importance of differences in the spike times between spike trains versus differences in spike count. For small $q, D$ is dominated by reliability (differences in spike count) and for large $q$ it is dominated by the precision (timing differences). The variability was averaged across different sets of attractor inputs to obtain $D_{a}^{\tau}\left(D_{a}^{t}\right)$ and across sets of surrogate inputs to obtain $D_{p}^{\tau}\left(D_{p}^{t}\right)$. The output spike trains were compared during interval (ii), between $750 \mathrm{~ms}$ and $1500 \mathrm{~ms}$ [Figs. 7(A)-7(F)]. The interval-based variability $D_{a}^{\tau}$ of the attractor response was approximately equal to $D_{p}^{\tau}$ over the entire range of $q$ values studied [Fig. 7(A)]. The rate of increase of $D_{a}^{\tau}$ with $q$ accelerated at $q \approx 0.01 \mathrm{~ms}^{-1}$. For these $q$ values, random jitter in the spike times across neurons re- 

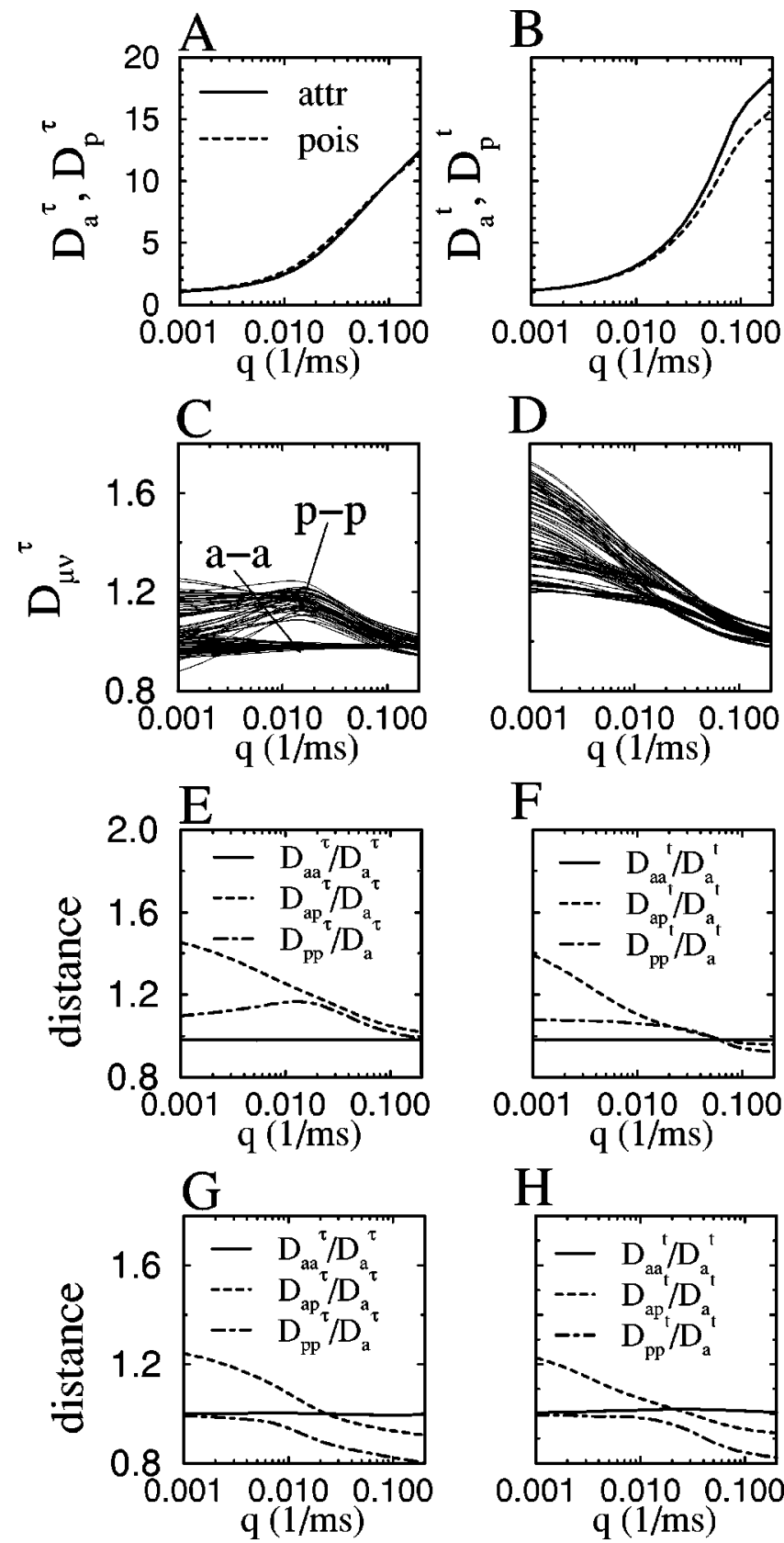

$\mathrm{H}$

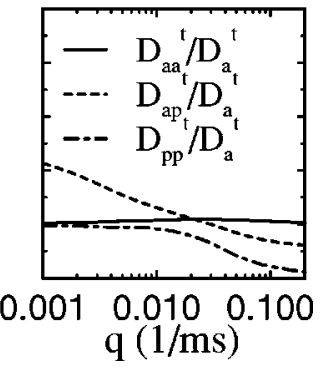

FIG. 7. Spike-metric analysis of the difference between attractor and surrogate responses. Mean variability of output spike trains in response to attractor spike trains ( $D_{a}^{\tau}$ and $D_{a}^{t}$, solid lines) and surrogate spike trains ( $D_{p}^{\tau}$ and $D_{p}^{t}$, dashed lines) for (A) the interval-based metric and (B) the time-based metric. For each pair of input sets the distance $D_{\nu \mu}$ is shown organized in three groups: $(\mathrm{C}, a a)$ two attractor responses, $(\mathrm{C}, p p)$ two surrogate responses, and (D) attractor and surrogate responses. The average distances for each group are shown: (E,G) $D_{a a}^{\tau}, D_{p p}^{\tau}, D_{p a}^{\tau}$ and $(\mathrm{F}, \mathrm{H})$ $D_{a a}^{t}, D_{p p}^{t}, D_{p a}^{t}$ for (E,F) interval (ii) and $(\mathrm{G}, \mathrm{H})$ interval (iii). The curves in $(\mathrm{C}),(\mathrm{E})$, and $(\mathrm{G})$ were normalized by $D_{a}^{\tau}$, those in (D), $(\mathrm{F})$, and $(\mathrm{H})$ were normalized by $D_{a}^{t}$. There were 20 sets of input spike trains, 10 were sets of attractor spike trains and 10 were sets of surrogate spike trains. The attractor spike trains were randomly chosen from the 500 trials in Fig. 4(Ad). For each set of inputs, 40 trials were generated. The metrics were calculated as described in Sec. II. ceiving the same inputs became the dominant contribution to the distance. The time-based variability behaved similarly, except that $D_{a}^{t}$ was larger than $D_{p}^{t}$ starting from $q$ $\approx 0.01 \mathrm{~ms}^{-1}$. The elements of $D_{\nu \mu}$ were divided in three groups, the distance between ( $a a$ ) two attractor responses, $(p p)$ two surrogate responses, and $(a p)$ an attractor and a surrogate response. Only different pairs were considered, hence there was no pa group. The individual curves $D_{\nu \mu}^{\tau}(q)$, normalized by $D_{a}^{\tau}$, for each pair of input sets are shown in Figs. 7(C) and 7(D). In this way the variability in the response to different realizations of input spike trains is expressed in terms of the variability due to intrinsic noise. The curves for $(a a)$ and $(p p)$ had a different shape. There was a large variability in the $(a p)$ curves. The means across all pairs, $D_{a a}^{\tau}\left(D_{a a}^{t}\right), D_{p p}^{\tau}\left(D_{p p}^{t}\right)$, and $D_{a p}^{\tau}\left(D_{a p}^{t}\right)$ are shown in Figs. 7(E) and 7(F) for the interval and time-based metrics, respectively. The variability $D_{a a}^{\tau}$ in attractor response to different input sets was approximately equal to the variability $D_{a}^{\tau}$ in the responses to one input set [Fig. 7(E)]. Thus, the attractor responses did not depend much on the actual realization of the set of input spike trains. This implies that the original spike trains of the first ensemble were similar. In contrast, the variability $D_{p p}^{\tau}$ in surrogate responses was significantly larger than either $D_{a}^{\tau}$ or $D_{p}^{\tau}$. For $q<0.01 \mathrm{~ms}^{-1}$, $D_{a p}^{\tau}$ was significantly larger than either $D_{a a}^{\tau}$ or $D_{p p}^{\tau}$. For $q$ $>0.01 \mathrm{~ms}^{-1}$, it converged to $D_{p p}^{\tau}$. Hence, attractor responses are more different from surrogate responses compared with the differences among either surrogate or attractor responses. The results for the time-based measures, $D_{a a}^{t}$, $D_{p p}^{t}$, and $D_{a p}^{t}$, were similar except that $D_{p p}^{t}$ and $D_{a p}^{t}$ were smaller than $D_{a a}^{t}$ for $q>0.02 \mathrm{~ms}^{-1}$. The analysis of the output spike trains during interval (iii) yielded similar results [Figs. $7(\mathrm{G})$ and $7(\mathrm{H})]$.

In summary, the difference between attractor and surrogate responses observed in Fig. 6 holds across different random realizations of the input spike trains.

\section{DISCUSSION}

In recent years the issue of reliability has been extensively studied both experimentally $[10-12,14,15,17,18]$ as well as theoretically $[9,13,38-43]$. An important motivation for studying reliability is that reliable spike trains might be more faithfully transmitted between cortical areas. However, very few studies [40] have explicitly addressed this issue. We show that the reliability and precision of an ensemble of neurons have a strong impact on downstream neuronal populations. In particular, the results presented here form a proof of principle that weak temporal modulations in the power of gamma-frequency oscillations in a given neural ensemble with only moderate changes in firing rate can strongly affect firing rate responses downstream via reliability. The basis for this effect was the switch induced by small changes in the gamma power between a chaotic state with unreliable spike trains and an entrained state with reliable spike trains. From a functional perspective it is better if the steady state of the first ensemble is chaotic, since the steady-state firing rate of the second ensemble would be low. In response to behavior- 
ally relevant stimuli, transient increases in the gamma power would then be able to increase the firing rate of the second ensemble. This is only feasible if the chaotic state is more probable in parameter space than the entrained state. For the model parameters studied here the current range $I_{A}$ for which chaotic spike trains are obtained is much smaller than the range for which entrainment is obtained. It remains for further investigation to determine what the relative size of chaotic versus entrained regimes is for more realistic driving stimuli.

The neurons in the second ensemble are driven by barrages of EPSPs. The reliability of the first ensemble determined the mean number of EPSPs in the barrages, whereas its precision (or jitter) determined the amplitude of the summed EPSPs. The higher the precision, the more coincident the EPSPs were, hence the higher the deflection of the postsynaptic membrane potential was. The entrainment of the first ensemble worked to increase the amplitude of the fluctuations in the synaptic drive. A neuron is in the balanced state when it receives approximately equal amounts of excitation and inhibition [44]. The firing rate of neurons in the balanced state is sensitive to the amplitude of the fluctuations, whereas when it is unbalanced the firing rate is mostly determined by the mean $[33,44-48]$. For the parameters used here, we found a range of driving currents $I_{0}=$ $-0.40-0.10 \mu \mathrm{A} / \mathrm{cm}^{2}$ where the neuron was in the balanced state. Changes in the reliability and precision of the input ensemble could in that case strongly modulate the neuron's firing rate.

The noise level in the two ensembles is a critical variable. For the purpose of this investigation intrinsic noise as well as background synaptic inputs were modeled as a white noise current with variance $2 D$ [33]. For the first ensemble it was $D=0.004 \mathrm{mV} / \mathrm{ms}^{2}$, whereas for the second ensemble it was $D=0.04 \mathrm{mV} / \mathrm{ms}^{2}$. The order of magnitude difference in $D$ values is significant. Due to the chaotic properties of the ensemble for $I=2.08 \mu \mathrm{A} / \mathrm{cm}^{2}$, a small dispersion in the initial conditions across different neurons quickly evolved into less reliable and precise spike trains. A similar phenomenon was observed using a periodically driven Fitzhugh-Nagumo model [42]. Hence, noise was not necessary to generate unreliability. However, when the driving current was increased to $I_{A}=2.3 \mu \mathrm{A} / \mathrm{cm}^{2}$, all neurons in the ensemble converged to exactly the same state (the same voltage $V$ and gating variables up to machine precision). When the current was then reduced to $I_{A}=2.08 \mu \mathrm{A} / \mathrm{cm}^{2}$ all neurons would be, and remain, in the same state-yielding reliable spike trains. To counteract this effect we added a weak noise term so that the neurons would be a little bit different during the phaselocked state. These small differences would then quickly increase upon entering the chaotic state.

For a higher level of depolarization, $I_{0}>0.2 \mu \mathrm{A} / \mathrm{cm}^{2}$, the neurons are periodically spiking in the absence of any periodic driving current $\left(I_{A}=0\right)$. In that case, we found that the neurons would phase lock to the periodic drive for almost all values of $I_{A}$ and $I_{0}$, and would fire $n$ spikes in $m$ cycles. However, the robustness of the phase locked state against intrinsic noise was different and decreased with the value of $m$. Hence, depending on the relative size of the amplitude of the driving current compared with the noise strength, phaselocking for $m=1$ would be stable, but would not be stable for $m=2$. A similar reliability resonance as a function of driving frequency was observed in experiments [13-15,17]. Hence the reliability and precision of the first ensemble could also be modulated by varying either frequency $f_{d}$ or driving current $I_{0}$ for sufficiently strong intrinsic noise.

An application of phase locking in olfactory computation was recently described by Hopfield and co-workers [49-51]. When many neurons have a similar firing rate, they become easily synchronized. Synchrony can be induced by weak synaptic coupling, or by a common oscillating drive to which the neurons phase lock. The latter case corresponds to the reliability resonance reported in Refs. [9,13-15,38]. This synchrony can be detected by downstream neurons, to perform the "many are equal" calculation [51].

For the purpose of analysis, spike trains obtained on multiple trials of a neuron are often considered as different realizations of the same underlying Poisson process with a timevarying firing rate [52]. However, spike trains produced by neurons driven by fluctuating stimulus wave forms form a nonrenewal process $[18,36]$. Hence, they do not satisfy Poisson statistics. We compared the response to attractor spike trains obtained from the model neuron to the response to surrogate spike trains with Poisson statistics. The surrogate spike trains were generated by randomly distributing the spikes in the original set across the neurons in the ensemble. When the synaptic connections between the two ensembles displayed STD, the neural response was significantly different. This is a biased comparison, since the surrogate spike trains differ in two aspects from the original spike trains: the intervals are independent (as are the spike times) and the distribution of intervals is exponential unlike the original spike trains. A more proper procedure would be to construct renewal spike trains with the same interval distribution as the original spike trains $[18,53]$. However, the implications of our manipulations are clear: the non-Poisson statistics of real spike trains is revealed in the presence of synaptic mechanisms that depend on the preceding sequence of interspike intervals. This means that the impact of a spike depends on the neuron that produced it, essentially turning each spike train into a labeled line [54]. It is not clear to what extent spike patterns are present in cortex under in vivo conditions and what their role in information processing is. However, preliminary analysis of data [8] obtained from cat lateral geniculate nucleus, a subcortical structure providing input the visual cortex, shows evidence for spike patterns [55].

We have investigated how the firing rate of a neuron is modulated by the precision and reliability of the ensemble of input neurons. The model system was not developed to represent a specific cortical area of a specific animal. Rather it was formulated to illustrate the general principle that reliability and precision can significantly affect downstream neuronal responses. However, we would also like to make the point that this general principle may be relevant to cortical information processing. To this purpose we need to justify how our model applies to the cortex. Our model is similar to recent work by Aertsen and co-workers [56], van Rossum and co-workers [57], and Brody and Hopfield [51]. 
Neither our model, nor the aforementioned models incorporate all experimentally observed properties of the cortical circuit and its constituent neurons. The physiology and anatomy of cortical circuits are still the subject of intense study and new data are emerging regularly. Besides, it would indeed be hard to identify what property is responsible for a specific functional behavior observed in very detailed and complex models. In our model we make the following assumptions: (1) The connections from the first ensemble to the second ensemble are excitatory and display short term depression; (2) the neurons only have a fast transient sodium current, a delayed rectifier potassium current, and a leak current; (3) there are $N=500$ neurons in an ensemble; (4) each neuron in the second ensemble receives inputs from $N_{\text {con }}$ $=100$ neurons of the first ensemble; (5) the neurons in an ensemble are not connected among themselves; (6) there are no feedback connections from the second ensemble to the first ensemble; (7) there are no inhibitory neurons. In the following we discuss these assumptions in relation to cortical circuits.

We could identify each ensemble with a different cortical area. The pyramidal cells of the superficial layer of the first cortical area project to cells in layer 4 of the second cortical area [29]. Cortical pyramidal cells are usually excitatory [29], hence this projection is presumably excitatory. Although STD has been observed for synapses between different layer 5 pyramidal cells [58] and synapses from layer 4 to layer $2 / 3$ pyramidal cells [32], it has not been observed for projections between different cortical areas. The lack of evidence for STD in this case is due to the difficulty of preserving the connections between two cortical areas in the cortical slice preparation and finding a pair of connected neurons in these two areas. The lack of evidence does, however, raise the issue of how important STD is for the results reported here. We obtained strong firing rate modulations with precision/reliability irrespective of whether the synapses had STD (Fig. 6) or not (Fig. 4). The non-Poisson structure of the spike trains generated by the first ensemble was evidenced as correlations between consecutive interspike intervals. To determine the potential impact of these correlations we looked for a biophysical mechanism that was sensitive to these correlations. STD is such a mechanism as well as spike timing dependent plasticity (see, for instance, Ref. [59]).

The nerve cell types in the cortex display a large number of different calcium and potassium channels [60] in addition to the fast transient sodium current and the delayed rectifier potassium current. These additional currents were not included in our model. This omission could have important consequences: these channels influence how the precision and reliability of the neurons in the first ensemble is affected by periodic drives, and they determine how the neurons in the second ensemble would respond. This is still a field of active research. We therefore elected to use a simple model that still had realistic spikes, rather than the popular leaky integrate-and-fire model $[20,30]$. We have, however, addressed the first issue in a recent paper [9].

The cortex has a columnar organization [29]. Neurons in the same cortical column have similar stimulus preferences and receive similar synaptic inputs. In our model, $N$ would correspond to the size of cortical column and $N_{\text {con }}$ to how many neurons of that cortical column project to the same neuron in the receiving cortical column. In a model for primary visual cortex a cortical column consisted of about a hundred neurons [61]. That number may reflect a trade-off between the small grain of the simulation and practical computability. The statistical properties for $N=500$ of the first ensemble were not much different from those for $N=1000$ (results not shown). Furthermore, similar results were obtained for a broad range of $N_{\text {con }}$ values [Figs. 5(C) and 5(D)]. This indicates that our results should be representative for a broad range of cortical column sizes. Note that cortical neurons typically receive more than a hundred inputs, recent work estimates that there are at least thousands of inputs [62]. These inputs were not explicitly included in the model, but they were represented as a stochastic noise current $C_{m} \eta$ (see the Methods).

There are extensive recurrent excitatory connections between nearby pyramidal cells in the cortex [29]. Our model does not incorporate these, but it is expected that the increases in firing rate that we observed would be amplified by recurrent excitation. Hence, it could only strengthen our results.

Regarding corticocortical connections Douglas and Martin write in Ref. [29]: The pattern that has emerged is that the pyramidal cells of the superficial layers project to middle layers (principally layer 4) of their target area, whereas the deep layer pyramidals project outside the middle layers to superficial and deep layers. These patterns have been used to classify patterns as feedforward (projecting to layer 4), or feedback (projecting outside layer 4). All cortical areas are reciprocally connected by these feedforward and feedback pathways. In the face of multiple parallel pathways projecting to and from cortical and subcortical areas these simple classifications of feedforward and feedback may not translate in functional significance. Here we studied the behavior of one pathway between two cortical areas in isolation. As the above quotation indicates, these pathways do not exist in isolation, rather they are part of a complicated network of cortical areas. The question then is how does a change in reliability and precision in one cortical area affect the rest of the interconnected cortical areas. This issue remains for further study.

We could also identify the ensembles as corresponding to different layers in the same cortical area. The cortical circuitry of area V1 in the macaque monkey has been reviewed by Callaway [63]. The picture is still incomplete, but support for a canonical cortical circuit diagram has emerged. There is a feedforward pathway going from the lateral geniculate nucleus to layer $4 \mathrm{C}$ proceeding on to layer $2-4 \mathrm{~B}$. There are also two feedback loops: one starting from and returning to layer $4 \mathrm{C}$ via layer 6 , and the other starting from and returning to layer $2-4 \mathrm{~B}$ via layer 5 . This is only a summary of the excitatory pathways. Approximately $20 \%$ of all cortical neurons are inhibitory [29]. Inhibitory neurons are thought to be important for controlling the timing of pyramidal cells [64$66]$. The inhibitory pathways are probably as complex as the excitatory ones. This raises the issue of how the firing rate is 
modulated by the reliability and precision of ensembles of inhibitory neurons. Preliminary results indicate that the firing rate of the neurons in the second ensemble is also strongly modulated by the precision (synchrony) of inhibitory inputs [67]. It is at present unclear how a circuit consisting of multiple excitatory and inhibitory loops would react to modulations of reliability and precision in one cortical layer. We plan to address this problem in a future study.

\section{ACKNOWLEDGMENTS}

I thank Jorge V. José and Peter J. Thomas for helpful comments on the manuscript.
[1] R. de Ruyter van Steveninck et al., Science 275, 1805 (1997).

[2] A. Warzecha and M. Egelhaaf, Science 283, 1927 (1999).

[3] D. Reich et al., J. Neurophysiol. 77, 2836 (1997).

[4] M. Berry, D. Warland, and M. Meister, Proc. Natl. Acad. Sci. U.S.A. 94, 5411 (1997).

[5] G. Buracas, A. Zador, M. DeWeese, and T. Albright, Neuron 20, 959 (1998).

[6] M. Berry and M. Meister, J. Neurosci. 18, 2200 (1998).

[7] P. Kara, P. Reinagel, and R. Reid, Neuron 27, 635 (2000).

[8] P. Reinagel and R. Reid, J. Neurosci. 22, 6837 (2002).

[9] S. Schreiber, J. Fellous, P. Tiesinga, and T. Sejnowski, J. Neurophysiol. 91, 194 (2004).

[10] H. Bryant and J. Segundo, J. Physiol. (London) 260, 279 (1976).

[11] Z. Mainen and T. Sejnowski, Science 268, 1503 (1995).

[12] L. Nowak, M. Sanchez-Vives, and D. McCormick, Cereb. Cortex 7, 487 (1997).

[13] J. Hunter, J. Milton, P. Thomas, and J. Cowan, J. Neurophysiol. 80, 1427 (1998).

[14] J.-M. Fellous et al., J. Neurophysiol. 85, 1782 (2001).

[15] U.a. Beierholm, J. Ryge, P. Alstrom, and O. Kiehn, J. Neurophysiol. 86, 1858 (2001).

[16] J. Haas and J. White, J. Neurophysiol. 88, 2422 (2002).

[17] J. Hunter and J. Milton, J. Neurophysiol. 90, 387 (2003).

[18] P. Tiesinga, J.-M. Fellous, and T. Sejnowski, Neural Comput. 14, 1629 (2002).

[19] N. Brenner et al., Neural Comput. 12, 1531 (2000).

[20] B. Knight, J. Gen. Physiol. 59, 767 (1972).

[21] E. Basar, C. Basar-Eroglu, S. Karakas, and M. Schurmann, Neurosci. Lett. 259, 165 (1999).

[22] E. Rodriguez et al., Nature (London) 397, 430 (1999).

[23] P. Tiesinga, J.-M. Fellous, J. José, and T. Sejnowski, Hippocampus 11, 251 (2001).

[24] P. Fries, J. Reynolds, A. Rorie, and R. Desimone, Science 291, 1560 (2001).

[25] A. Engel, P. Fries, and W. Singer, Nat. Rev. Neurosci 2, 704 (2001).

[26] J. Brumberg, Neuroscience 114, 239 (2002).

[27] P. Tiesinga and J. José, Network 11, 1 (2000).

[28] X. Wang and G. Buzsáki, J. Neurosci. 16, 6402 (1996).

[29] G. Shepherd, Synaptic Organization of the Brain, 4th ed. (Oxford University Press, Oxford, 1998).

[30] C. Koch, Biophysics of Computation (Oxford University Press, New York, 1999).

[31] M. Goldman, P. Maldonado, and L. Abbott, J. Neurosci. 22, 584 (2002).

[32] L. Abbott, J. Varela, K. Sen, and S. Nelson, Science 275, 220 (1997).
[33] P. Tiesinga, J. José, and T. Sejnowski, Phys. Rev. E 62, 8413 (2000).

[34] P. Tiesinga and T. Sejnowski, Neural Comput. 16, 251 (2004).

[35] J. Victor and K. Purpura, J. Neurophysiol. 76, 1310 (1996).

[36] D. Reich, J. Victor, and B. Knight, J. Neurosci. 18, 10090 (1998).

[37] S. Strogatz, Nonlinear Dynamics and Chaos (Addison-Wesley, Reading, MA, 1994).

[38] P. Tiesinga, Phys. Rev. E 65, 041913 (2002).

[39] T. Shimokawa, A. Rogel, K. Pakdaman, and S. Sato, Phys. Rev. E 59, 3461 (1999).

[40] G. Cecchi et al., Proc. Natl. Acad. Sci. U.S.A. 97, 5557 (2000).

[41] R. Brette and E. Guigon, Neural Comput. 15, 279 (2003).

[42] E. Kosmidis and K. Pakdaman, J. Comput. Neurosci. 14, 5 (2003).

[43] B. Gutkin, G. Ermentrout, and M. Rudolph, J. Comput. Neurosci. 15, 91 (2003).

[44] E. Salinas and T. Sejnowski, J. Neurosci. 20, 6193 (2000).

[45] F. Chance, L. Abbott, and A. Reyes, Neuron 35, 773 (2002).

[46] A. Burkitt, Biol. Cybern. 85, 247 (2001).

[47] K. Miller and T. Troyer, J. Neurophysiol. 87, 653 (2002).

[48] D. Hansel and C. van Vreeswijk, J. Neurosci. 22, 5118 (2002).

[49] J. Hopfield, Nature (London) 376, 33 (1995).

[50] J. Hopfield and C. Brody, Proc. Natl. Acad. Sci. U.S.A. 98, 1282 (2001).

[51] C. Brody and J. Hopfield, Neuron 37, 843 (2003).

[52] P. Dayan and L. Abbott, Theoretical Neuroscience (MIT Press, Cambridge, MA, 2001).

[53] M. Oram, M. Wiener, R. Lestienne, and B. Richmond, J. Neurophysiol. 81, 3021 (1999).

[54] D. Reich, F. Mechler, and J. Victor, Science 294, 2566 (2001).

[55] J.-M. Fellous, P. Tiesinga, P. Thomas, and T. Sejnowski, J. Neurosci. (to be published).

[56] M. Diesmann, M. Gewaltig, and A. Aertsen, Nature (London) 402, 529 (1999).

[57] M. van Rossum, G. Turrigiano, and S. Nelson, J. Neurosci. 22, 1956 (2002).

[58] M. Tsodyks and H. Markram, Proc. Natl. Acad. Sci. U.S.A. 94, 719 (1997).

[59] S. Song, K. Miller, and L. Abbott, Nat. Neurosci. 3, 919 (2000).

[60] B. Hille, Ion Channels of Excitable Membranes, 3rd ed. (Sinauer Associates, Sunderland, MA, 2001).

[61] D. Somers, S. Nelson, and M. Sur, J. Neurosci. 15, 5448 (1995).

[62] D. Pare and A. Destexhe, J. Neurophysiol. 81, 1531 (1999).

[63] E. Callaway, Annu. Rev. Neurosci. 21, 47 (1998). 
[64] M. Galarreta and S. Hestrin, Science 292, 2295 (2001).

[65] V. Gauck and D. Jaeger, J. Neurosci. 20, 3006 (2000).

[66] P. Tiesinga, J.-M. Fellous, J. José, and T. Sejnowski, Network 13, 41 (2002).
[67] J. Jose et al., Abstr. Soc. Neurosci. 28, 55.6 (2002).

[68] We used the FORTRAN code provided by J. D. Victor on the web page http://www-users.med.cornell.edu/ $\sim$ jdvicto/metricdf.html to calculate the metrics. 